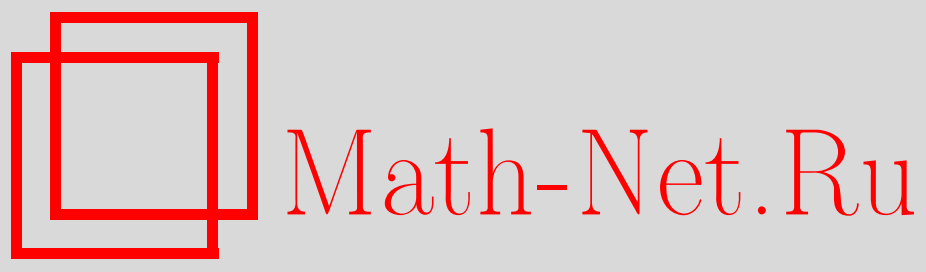

Л. П. Нижник, Одномерный оператор Шрёдингера с точечными взаимодействиями в пространствах Соболева, Функи. анализ и его прил., 2006, том 40, выпуск $2,74-79$

DOI: https://doi.org/10.4213/faa9

Использование Общероссийского математического портала Math-Net.Ru подразумевает, что вы прочитали и согласны с пользовательским соглашением

http://www . mathnet.ru/rus/agreement

Параметры загрузки:

IP: 3.89.197.203

26 апреля 2023 г., 14:36:35

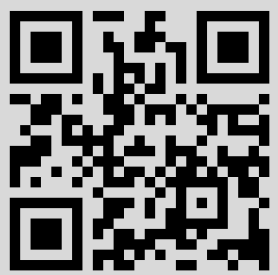




\title{
Одномерный оператор Шрёдингера с точечными взаимодействиями в пространствах Соболева
}

\author{
(c) 2006. Л. П. Нижник
}

Обычно самосопряженные операторы Шрёдингера изучаются в пространствах $L_{2}\left(\mathbb{R}^{d}\right)$. Однако ряд специальных вопросов требует рассмотрения операторов Шрёдингера в пространствах Соболева $W_{2}^{m}\left(\mathbb{R}^{d}\right)$. Идея изучения операторов в пространствах, отличных от $L_{2}\left(\mathbb{R}^{d}\right)$, не нова. Различные проблемы анализа, дифференциальных уравнений и математической физики способствовали созданию и изучению операторов как в абстрактных шкалах гильбертовых пространств, так и в шкалах пространств Соболева [1-5]. Однако спектральная теория дифференциальных операторов в пространствах Соболева практически не разработана, несмотря на ее содержательность и возможные приложения [6].

1. Задача Штурма-Лиувилля с производными высоких порядков в граничных условиях приводит к самосопряженным операторам в пространствах Соболева. Так, задача $-y^{\prime \prime}=\lambda y$ на интервале $0<x<\pi$ с граничными условиями $y^{\prime \prime}(0)-y(0)=0, y^{\prime \prime}(\pi)-y(\pi)=0$ является самосопряженной в пространстве Соболева $W_{2}^{1}(0, \pi)$ с собственными функциями $u_{n}=\sin n x, n=1,2, \ldots$, и $u^{ \pm}=$ $\exp ( \pm x)$. Эти функции образуют полную ортогональную систему в пространстве $W_{2}^{1}(0, \pi)$. Если рассматривать граничные условия в виде $y^{\prime \prime}(0)+y(0)=0$, $y^{\prime \prime}(\pi)+y(\pi)=0$, то задача на собственные значения будет уже самосопряженной относительно индефинитного скалярного произведения в $W_{2}^{1}(0, \pi)$, задаваемого равенством $[u, v]=\left(u^{\prime}, v^{\prime}\right)_{L_{2}}-(u, v)_{L_{2}}$.

2. Свободный оператор Шрёдингера, $-\Delta$, является самосопряженным как в пространстве $L_{2}\left(\mathbb{R}^{d}\right)$, так и в пространствах Соболева $W_{2}^{m}\left(\mathbb{R}^{d}\right)$. Одним из способов задания операторов Шрёдингера с точечными взаимодействиями в точке $x_{0}$ является рассмотрение самосопряженных расширений минимального симметрического оператора, определенного на $C_{0}^{\infty}\left(\mathbb{R}^{d} \backslash\left\{x_{0}\right\}\right)$ выражением $L_{\min } \varphi=-\Delta \varphi$ [7-9]. Так определенный оператор $L_{\min }$ является также симметрическим оператором в пространстве Соболева $W_{2}^{m}\left(\mathbb{R}^{d}\right)$ с равными индексами дефекта. Для целых $m \geqslant 0$ индексы дефекта $i(m, d)$ равны количеству всех целочисленных мультииндексов $\alpha=\left(\alpha_{1}, \ldots, \alpha_{d}\right)$, удовлетворяющих условию $|\alpha|=\alpha_{1}+\cdots+\alpha_{d}<2+m-\frac{1}{2} d[10]$. В пространстве $L_{2}\left(\mathbb{R}^{d}\right)$ (т. е. при $\left.m=0\right)$ это приводит к хорошо известному факту, что индексы дефекта $i(0, d)$ отличны от нуля лишь в размерностях $d=1,2,3$ и равны соответственно $2,1,1$.

Для важных размерностей $d=1,2,3$ индексы дефекта определяются формулами $i(m, 1)=m+2, i(m, 2)=\frac{1}{2}(m+1)(m+2), i(m, 3)=\frac{1}{6}(m+1)(m+2)(m+3)$.

Несмотря на то что оператор $L_{\min }$ в пространствах $W_{2}^{m}\left(\mathbb{R}^{d}\right)$ не плотно задан (он плотно определен лишь при $m=0$ или при $d \geqslant 2+2 m$ ), можно конструктивно описать все его самосопряженные расширения. Это приводит к большому разнообразию самосопряженных операторов Шрёдингера с точечными взаимодействиями в пространствах Соболева $W_{2}^{m}\left(\mathbb{R}^{d}\right)$, в частности, описывающих суперсингулярные сферически несимметрические точечные взаимодействия [10]. 
3. Для изучения одномерных операторов Шрёдингера в пространствах Соболева нам понадобятся следующие вспомогательные факты. Будем обозначать через $u_{r}=\frac{1}{2}[u(+0)+u(-0)]$ и $u_{s}=u(+0)-u(-0)$ среднее значение и скачок функции $u$ в точке $x=0$ соответственно. Для функций $u \in W_{2}^{2 m+2}\left(\mathbb{R}^{1} \backslash\{0\}\right)$ введем обозначение для квазипроизводных при $x \neq 0: u^{[2 k]}=\left(-D^{2}+1\right)^{k} u$, $u^{[2 k+1]}=D u^{[2 k]}$, где $D=d / d x$, а через $\Gamma_{1} u$ и $\Gamma_{2} u$ обозначим векторы граничных данных до порядка $2 m+1$ :

$$
\Gamma_{1} u=\left(u_{r}, \ldots,(-1)^{k} u_{r}^{[k]}, \ldots,-u_{r}^{[2 m+1]}\right), \quad \Gamma_{2} u=\left(u_{s}^{[2 m+1]}, \ldots, u_{s}^{[k]}, \ldots, u_{s}\right) .
$$

Тогда для функций $u, v \in W_{2}^{2 m+2}\left(\mathbb{R}^{1} \backslash\{0\}\right)$ справедлива формула Грина

$$
\left(u^{[2 m+2]}, v\right)_{L_{2}}-\left(u, v^{[2 m+2]}\right)_{L_{2}}=\Gamma_{2} u \cdot \Gamma_{1} v-\Gamma_{1} u \cdot \Gamma_{2} v,
$$

где точка обозначает скалярное произведение в евклидовом пространстве $E^{2 m+2}$.

Формула Грина $(1)$ показывает, что $\left(E^{2 m+2}, \Gamma_{1}, \Gamma_{2}\right)$ является граничной тройкой, или пространством граничных значений (ПГЗ) [11-13], для минимального оператора $\left(-D^{2}+1\right)^{m+1} \uparrow_{C_{0}^{\infty}\left(\mathbb{R}^{1} \backslash\{0\}\right)}$ в пространстве $L_{2}\left(\mathbb{R}^{1}\right)$.

Наряду с самосопряженным в пространстве $W_{2}^{m}\left(\mathbb{R}^{1}\right)$ оператором $L=-D^{2}+1$ с областью определения $\mathscr{D}(L)=W_{2}^{m+2}\left(\mathbb{R}^{1}\right)$ рассмотрим также в $W_{2}^{m}\left(\mathbb{R}^{1}\right)$ оператор $L^{(m)}$ с областью определения $\mathscr{D}\left(L^{(m)}\right)=W_{2}^{m}\left(\mathbb{R}^{1}\right) \cap W_{2}^{2 m+2}\left(\mathbb{R}^{1} \backslash\{0\}\right)$ и законом действия $L^{(m)} u=\left(-D^{2}+1\right)^{-m} u^{[2 m+2]}$. Ясно, что $L^{(m)}$ совпадает с $L$ на $W_{2}^{2 m+2}\left(\mathbb{R}^{1}\right)$. Оператор $L^{(m)}$ для неплотно заданного оператора $L_{\min }$ заменяет отсутствующий оператор $L_{\min }^{*}$, играющий ключевую роль в теории самосопряженных расширений.

Будем здесь и в дальнейшем определять скалярное произведение в пространстве $W_{2}^{m}\left(\mathbb{R}^{1}\right)$ так, что $(u, v)_{W_{2}^{m}}=\left(\left(-D^{2}+1\right)^{m} u, v\right)$ для $u, v \in \mathbb{C}_{0}^{\infty}\left(\mathbb{R}^{1}\right)$. Для функций из пространства $W_{2}^{m}\left(\mathbb{R}^{1}\right) \cap W_{2}^{2 m+2}\left(\mathbb{R}^{1} \backslash\{0\}\right)$ формула Грина (1) принимает вид

$$
\left(L^{(m)} u, v\right)_{W_{2}^{m}}-\left(u, L^{(m)} v\right)_{W_{2}^{m}}=\Gamma_{2}^{(m)} u \cdot \Gamma_{1}^{(m)} v-\Gamma_{1}^{(m)} u \cdot \Gamma_{2}^{(m)} v,
$$

где векторы граничных данных $\Gamma_{1}^{(m)} u, \Gamma_{2}^{(m)} u$ определяются равенствами

$$
\Gamma_{2}^{(m)} u=\left(u_{s}^{[2 m+1]}, \ldots, u_{s}^{[m]}\right), \quad \Gamma_{1}^{(m)} u=\left(u_{r}, \ldots,(-1)^{k} u_{r}^{[k]}, \ldots,(-1)^{(m+1)} u_{r}^{[m+1]}\right) .
$$

Если $B$ - самосопряженный оператор в евклидовом пространстве $E^{m+2}$, то граничные условия

$$
\Gamma_{2}^{(m)} u=B \Gamma_{1}^{(m)} u
$$

являются самосопряженными в пространстве $W_{2}^{m}\left(\mathbb{R}^{1}\right)$. Сужение оператора $L^{(m)}$ на все функции, удовлетворяющие граничному условию (3), будем обозначать через $L_{B}$ и называть оператором Шрёдингера с точечным взаимодействием в точке $x_{0}=0$, описываемым граничными условиями (3). Граничные условия (3) будем называть полными, если не существует нетривиальной функции из дефектного подпространства $N$ оператора $L_{\min }$ в пространстве $W_{2}^{m}\left(\mathbb{R}^{1}\right)$, удовлетворяющей этому граничному условию. 
Tеорема 1. Пусть самосопряжсенный оператор $B$ в пространстве $E^{m+2}$ задает полные граничные условия (3). Тогда оператор $L_{B}$ является существенно самосопряженным ограниченно обратимым оператором в пространстве $W_{2}^{m}\left(\mathbb{R}^{1}\right)$.

ЗАмечание 1. Граничные условия (3) описывают лишь обратимые самосопряженные в пространстве $W_{2}^{m}\left(\mathbb{R}^{1}\right)$ расширения оператора $L_{\min }$. Для описания всех самосопряженных расширений можно, например, условия (3) заменить на $(I-U) \Gamma_{2}^{(m)} u=i(I+U) \Gamma_{1}^{(m)} u$, где $U-$ унитарный оператор в пространстве $E^{m+2}$. Отметим новизну конструкции квази-ПГЗ в формуле $(2)$ для неплотно заданного в пространстве $W_{2}^{m}\left(\mathbb{R}^{1}\right)$ оператора $L_{\min }$ через обычное ПГЗ для степеней $\left(L_{\min }^{*}\right)^{m}$ в пространстве $L_{2}\left(\mathbb{R}^{1}\right)$.

4. Для описания самосопряженных расширений в пространстве Соболева $W_{2}^{m}\left(\mathbb{R}^{1}\right)$ минимального симметрического оператора $L_{\min }$ можно также воспользоваться абстрактной теорией самосопряженных расширений неплотно заданных операторов [11-17]. Не привлекая понятия отношения, естественного для теории расширений неплотно заданного симметрического оператора $[16,17]$, воспользуемся классической теорией Бирмана-Крейна-Вишика, справедливой и в этом случае. Действительно, пусть $A$ - строго положительный самосопряженный оператор в гильбертовом пространстве $H$, а $N \subset H$ - замкнутое подпространство, которое можно рассматривать как дефектное подпространство симметрического оператора $A_{N}=A\left\lceil_{\mathscr{D}\left(A_{N}\right)}\right.$, где $\mathscr{D}\left(A_{N}\right)=\{\varphi: \varphi \in \mathscr{D}(A),(A \varphi, \eta)=0$ для любого $\eta \in N\}$. Самосопряженные ограниченно обратимые расширения $A_{N, B}$ оператора $A_{N}$ можно параметризовать ограниченными самосопряженными операторами $B$ в подпространстве $N$. Справедливы формулы $\mathscr{D}\left(A_{N, B}\right)=$ $\left\{\psi: \psi=\varphi+B P_{N} A \varphi\right.$ для любого $\left.\varphi \in \mathscr{D}(A)\right\}$, где $P_{N}$ - ортопроектор в $H$ на $N$, т. е. $A_{N, B} \psi=A_{N, B}\left(\varphi+B P_{N} A \varphi\right)=A \varphi, A_{N, B}^{-1}=A^{-1}+B P_{N}$. Единственное дополнительное условие на оператор $B$ - тривиальная разрешимость уравнения $\varphi+B P_{N} A \varphi=0$ на множестве $N \cap \mathscr{D}(A)$. Это обеспечивает корректность приведенной выше конструкции. В случае, когда $N \cap \mathscr{D}(A)=\{0\}$, и только в этом случае оператор $A_{N}$ плотно определен в пространстве $H$, а дополнительное условие на оператор $B$ выполняется автоматически.

Пусть теперь $N \cap \mathscr{D}(A) \neq\{0\}$, но $N \cap \mathscr{D}\left(A^{m}\right)=\{0\}$ при некотором $m>1$. Тогда на прямой сумме $\mathscr{L}_{m}=\mathscr{D}\left(A^{m}\right) \dot{+} N$ определены косые проекторы $R^{(m)}$ на $\mathscr{D}\left(A^{m}\right)$ и $S^{(m)}$ на $N\left(R^{(m)}+S^{(m)}=I\right)$ и оператор $A_{N}^{(m)}=A R^{(m)}$. Ясно, что $\operatorname{Ker}\left(A_{N}^{(m)}\right)=N$, а операторы $A_{N}^{(m)}$ и $A$ совпадают на $\mathscr{D}\left(A^{m}\right)$. Справедлив абстрактный аналог формулы Грина $(2)$ :

$$
\left(A_{N}^{(m)} u, v\right)_{H}-\left(u, A_{N}^{(m)} v\right)_{H}=\left(\Gamma_{2}^{(m)} u, \Gamma_{1}^{(m)} v\right)_{N}-\left(\Gamma_{1}^{(m)} u, \Gamma_{2}^{(m)} v\right)_{N}, \quad u, v \in \mathscr{L}_{m}
$$

где $\Gamma_{2}^{(m)}=S^{(m)}$, а $\Gamma_{1}^{(m)}=-P_{N} A R^{(m)}$, задающий квази-ПГЗ для неплотно заданного симметрического оператора $A_{N}$.

Определим теперь симметрический оператор $A_{N, B}^{(m)}$ как сужение оператора $A_{N}^{(m)}$ на все элементы $u$, удовлетворяющие граничному условию $\Gamma_{2}^{(m)} u+$ $B \Gamma_{1}^{(m)} u=0$ с заданным ограниченным самосопряженным оператором $B$ в подпространстве $N$. 
ТЕОрема 2. Пусть ограниченный самосопряэсенный оператор В удовлетворяет упомянутому выше дополнителъному условию. Тогда оператор $A_{N, B}^{(m)}$ является существенно самосопряженным в пространстве $H$, а его замыкание совпадает с оператором $A_{N, B}$.

Для эффективного описания дефектного подпространства $N$ и самосопряженных расширений оператора $L_{\min }$ в пространстве $W_{2}^{m}\left(\mathbb{R}^{1}\right)$ используются фундаментальные решения $E_{2 m}(x)$ уравнения $\left(-D^{2}+1\right)^{m} E_{2 m}(x)=\delta$ и их производные $E_{2 m-1}(x)=E_{2 m}^{\prime}(x)$. Явный вид фундаментальных решений легко получить, используя преобразование Фурье:

$$
\begin{aligned}
E_{2 m}(x) & =\frac{1}{(m-1) ! 2^{m}} \sum_{k=0}^{m-1} C_{2 m-2-k}^{k}(2 m-3-2 k) ! !|x|^{k} \exp (-|x|), \\
E_{2 m+1}(x) & =-\frac{x}{2 m} E_{2 m}(x), \quad m \geqslant 1 .
\end{aligned}
$$

Приведем явный вид первых четырех функций $E_{k}$ :

$$
\begin{array}{ll}
E_{1}(x)=-\frac{\operatorname{sign} x}{2} \exp (-|x|), & E_{2}(x)=\frac{1}{2} \exp (-|x|), \\
E_{3}(x)=-\frac{x}{4} \exp (-|x|), & E_{4}(x)=\frac{1}{4}(1+|x|) \exp (-|x|) .
\end{array}
$$

ЛЕмма 1. Совокупность фундаментальных решений $E_{j}, j=m+1, \ldots$, $2 m+2$, образует базис в дефектном подпространстве $N$ оператора $L_{\min } \theta$ пространстве $W_{2}^{m}\left(\mathbb{R}^{1}\right)$.

Это явное описание дефектного подпространства $N$ оператора $L_{\min }$ и простой закон действия $L E_{j}=E_{j-2}$ оператора $L$ на указанном базисе позволяют явно связать конструкции операторов Шрёдингера с точечными взаимодействиями на основе граничных условий (3) и на основе самосопряженных расширений оператора $L_{\min }$. В частности, например, для пространства $W_{2}^{1}\left(\mathbb{R}^{1}\right)$ дополнительное условие на оператор $B$ имеет вид неравенства $B E_{2}+E_{4} \neq 0$.

5. Приведем еще способ задания операторов Шрёдингера с точечным взаимодействием в точке $x=0$ в пространстве $W_{2}^{m}\left(\mathbb{R}^{1}\right)$ с помощью сингулярных возмущений. Для этого рассмотрим формулу Грина $(2)$ для $v \in W_{2}^{2 m+2}\left(\mathbb{R}^{1}\right)$ : $(u, L v)_{W_{2}^{m}}=\left(L^{(m)} u, v\right)_{W_{2}^{m}}-\Gamma_{2}^{(m)} u \cdot \Gamma_{1}^{(m)} v$. Она естественно определяет оператор $L^{+}$на $\mathscr{D}\left(L^{(m)}\right)$, действующий по правилу

$$
L^{+} u=L^{(m)} u-\sum_{k=0}^{m+1} u_{s}^{[2 m+1-k]} \delta^{[k]},
$$

где $\delta^{[k]}$ - квазипроизводные от $\delta$-функции Дирака, определенные равенством $\left(\delta^{[k]}, v\right)=(-1)^{k} v_{r}^{[k]}$.

Формула (5) является аналогом известного правила, связывающего обобщенное дифференцирование разрывных функций с их поточечным дифференцированием. В силу этого оператор $L^{(m)}$ в работе [10] назван поточечным применением в $W_{2}^{m}$ оператора $L=-D^{2}+1$.

$\mathrm{C}$ помощью представления (5) и граничных условий (3) доказывается 
Tеорема 3. Oператор $L_{B}$ из теоремы 1 можно представить в виде

$$
L_{B} u=L^{+} u+\sum_{i, j=0}^{m+1} b_{i j} \delta^{[i]}\left(u, \delta^{[j]}\right),
$$

где область определения оператора $L_{B}$ состоит из всех функиий $u \in W_{2}^{m}\left(\mathbb{R}^{1}\right) \cap$ $W_{2}^{2 m+2}\left(\mathbb{R}^{1} \backslash\{0\}\right)$, для которых правая часть в (6) принадлежит $W_{2}^{m}\left(\mathbb{R}^{1}\right)$, а на таких функциях $L_{B} u=L^{(m)} u=\left(-D^{2}+1\right)^{-m} u^{[2 m+2]}$.

6. Рассмотрим одномерный оператор Шрёдингера $H_{n}=-d^{2} / d x^{2}+\alpha \delta^{(n)}(x)$, потенциалом в котором является $n$-я производная от $\delta$-функции Дирака. Для определения действия оператора $H_{n}$ на разрывные функции $\psi(x) \in W_{2}^{2+n}\left(\mathbb{R}^{1} \backslash\right.$ $\{0\})$ необходимо определить $-d^{2} \psi(x) / d x^{2}$ и произведение $\delta^{(n)}(x) \cdot \psi(x)$. В случае пространства $L_{2}\left(\mathbb{R}^{1}\right)$ эти выражения можно понимать в смысле теории обобщенных функций:

$-\frac{d^{2}}{d x^{2}} \psi(x)=-\psi^{\prime \prime}(x)-\psi_{s} \delta^{\prime}-\psi_{s}^{\prime} \delta, \quad \delta^{(n)} \cdot \psi(x)=\sum_{k=0}^{n} C_{n}^{k} \psi_{r}^{(n-k)}(-1)^{n-k} \delta^{(k)}(x)$.

В последнем выражении выбрано одно из «естественных» продолжений функционала $\delta^{(n)}$ на разрывные пробные функции $\varphi$ с помощью равенства $\left(\delta^{(n)}, \varphi\right)=$ $(-1)^{n} \varphi_{r}^{(n)}[9]$. С учетом этого обстоятельства условие $H_{n} \psi \in L_{2}\left(\mathbb{R}^{1}\right)$ приводит к известным [18] граничным условиям

$$
\psi_{s}^{\prime}=(-1)^{n} \alpha \psi_{r}^{(n)}, \quad \psi_{s}=(-1)^{(n-1)} \alpha n \psi_{r}^{(n-1)} .
$$

Кроме того, при $n \geqslant 2$ выполняются условия $\psi_{r}^{(j)}=0, j=0, \ldots, n-2$. Для $n=$ 0,1 граничные условия (7) при вещественных $\alpha$ определяют самосопряженный в $L_{2}\left(\mathbb{R}^{1}\right)$ оператор Шрёдингера с $\alpha \delta$ - и $\alpha \delta^{\prime}$-потенциалами. Граничные условия $\psi_{s}^{\prime}=-\alpha \psi_{r}^{\prime}, \psi_{s}=\alpha \psi_{r}$ для $\alpha \delta^{\prime}$-потенциала отличаются от граничных условий $\psi_{s}^{\prime}=0, \psi_{s}=\alpha \psi_{r}^{\prime}$, определяющих оператор Шрёдингера с $\delta^{\prime}$-взаимодействием интенсивности $\alpha[8,9,19]$. При $n \geqslant 2$ граничные условия $(7)$ не определяют самосопряженных операторов в пространстве $L_{2}\left(\mathbb{R}^{1}\right)$. Однако выражению $H_{n}$ при $n \geqslant 2$ можно придать смысл самосопряженного оператора в пространстве $W_{2}^{m}\left(\mathbb{R}^{1}\right)$ при $m \geqslant n-1$, если положить

$$
\left(H_{n}+1\right) \psi=L^{+} \psi+\sum_{k=0}^{n} C_{n}^{k} \psi_{r}^{(n-k)}(-1)^{(n-k)} \delta^{(k)}
$$

и найти область определения оператора $H_{n}$ из условия принадлежности функции $\psi$ и правой части равенства (8) пространству $W_{2}^{m}\left(\mathbb{R}^{1}\right)$. Это дает граничные условия для оператора $H_{n}$ в пространстве $W_{2}^{m}\left(\mathbb{R}^{1}\right)$ вида $(3)$ со специальной матрицей $B$. В частности, оператор $H_{2}=-d^{2} / d x^{2}+\alpha \delta^{\prime \prime}$ является самосопряженным в пространстве $W_{2}^{1}\left(\mathbb{R}^{1}\right)$, а функции из области его определения удовлетворяют граничным условиям $\psi_{s}^{\prime}+\alpha \psi_{r}=\psi_{s}^{\prime \prime}-2 \alpha \psi_{r}^{\prime}=\psi_{s}^{\prime \prime \prime}+2 \alpha \psi_{r}=0$. Возможны и другие интерпретации оператора $H_{n}$ в пространствах, более широких, чем $W_{2}^{m}\left(\mathbb{R}^{1}\right)$ [4].

ЗАмечАние 2. Выше в пространствах Соболева рассмотрен оператор Шрёдингера с одноточечным взаимодействием. Переход к многоточечным взаимодействиям аналогичен соответствующему переходу в случае пространства $L_{2}\left(\mathbb{R}^{1}\right)$. При этом возникает нетривиальная задача об определении количества 
отрицательных собственных значений для оператора Шрёдингера с многоточечными взаимодействиями. В этом направлении ряд точных результатов получен B $[20,21]$.

ЗАМЕчАНИЕ 3. Простейшей физической задачей, приводящей к оператору Шрёдингера с точечным взаимодействием в пространстве $W_{2}^{1}\left(\mathbb{R}^{1}\right)$, является задача о собственных поперечных колебаниях струны, расположенной на оси $O X$, с точечной массой в начале координат, на которую действует внешняя сила, пропорциональная отклонению массы от положения равновесия [10]. Если внешняя сила отсутствует, то задача сводится к самосопряженному в $L_{2}\left(\mathbb{R}^{1}\right)$ оператору Шрёдингера с $\delta^{\prime}$-взаимодействием [10, 19].

\section{ЛиТЕРАТУРА}

1. Berezansky $Y u$. Expansion in Eigenfunction of Self-Adjoint Operators. Amer. Math. Soc., Providence, RI, 1968. 2. Березанский Ю. М. В кн.: Спектральный анализ дифференциальных операторов. Институт математики АН УССР, Киев, 1980. 3. Berezansky Yu., Brasche, J. Methods Funct. Anal. Topology, 8, No. 4, 1-14 (2002). 4. Kurasov P. Integral Equ. Oper. Theory, 45, No. 4, 437-460 (2003). 5. Nizhnik L. P. Methods Funct. Anal. Topology, 7, No. 1, 76-81 (2001). 6. Шкаликов А. А. Труды семин. Петровского, 9, 190-229 (1983). 7. Березин Ф. А., Фаддеев Л. Д. Докл. АН CCCP, 137, 1011-1014 (1961). 8. Albeverio S., Gesztesy F., Høegh-Krohn R., Holden H. Solvable Models in Quantum Mechanics. Springer-Verlag, Berlin, 1988. 9. Albeverio S., Kurasov P. Singular Perturbations of Differential Operators and Solvable Schrödinger Type Operators. Cambridge University Press, 2000. 10. Albeverio S., Nizhnik L. Lett. Math. Phys., 70, 185-194 (2004). 11. Derkach, V. A., Malamud, M. M. J. Funct. Anal., 95, 1-95 (1991). 12. Gorbachuk V. I., Gorbachuk M. L. Boundary Value Problems for Operator Differential Equations. Kluwer Academic Publishers, 1991. 13. Kuzhel S., Kuzhel A. Regular Extensions of Hermitian Operators. VSP, Utrecht, 1998. 14. Красносельский М. А. Укр. матем. ж., 1, 21-38 (1949). 15. Крейн М. Матем. сб., 20, 21, 431-495, 365-404 (1947). 16. Кочубей А. Н. Сиб. матем. ж., 18, №2, 314-320 (1977). 17. Маламуд M. Укр. матем. ж., 44, 1658-1688 (1992). 18. Griffiths D. J. J. Phys. A: Math. Gen., 26, $2265-2267$ (1993). 19. Nizhnik L. P. J. Funct. Anal. Appl., 37, No. 1, 85-88 (2003). 20. Albeverio S., Nizhnik L. Methods Funct. Anal. Topology, 9, 273-286 (2003). 21. Albeverio S., Nizhnik L. Lett. Math. Phys., 65, 27-35 (2003).

Институт математики НАН Украины e-mail: nizhnik@imath.kiev.ua
Поступило в редакцию 13 октября 2004 г. 